\title{
Fatores socioambientais associados à ocorrência de quedas em idosos
}

\author{
Social and environmental factors \\ associated with the occurrence of falls in the elderly
}

\author{
Gustavo Nunes Pereira ${ }^{1}$ \\ Patricia Morsch ${ }^{1}$ \\ Diene Gomes Colvara Lopes ${ }^{1}$ \\ Margarete Diprat Trevisan ${ }^{1}$ \\ André Ribeiro ${ }^{1}$ \\ Joel Hirtz do Nascimento Navarro ${ }^{1}$ \\ Denielli da Silva Gonçalves Bós ${ }^{1}$ \\ Miriam Souza dos Santos Vianna ${ }^{1}$ \\ Ângelo José Gonçalves Bós ${ }^{1}$
}

${ }^{1}$ Instituto de Geriatria e Gerontologia, PUCRS. Av. Ipiranga 6681, Partenon. 90.619-900 Porto Alegre RS Brasil.

gustavojaguarao@hotmail.com

\begin{abstract}
This article seeks to investigate environmental aspects that may be associated with falls among elderly residents in the state of Rio Grande do Sul. It is a population-based, descriptive, crosssectional study, with primary data collection taken from an epidemiological study entitled A Profile of the Elderly in the state of Rio Grande do Sul. The study was conducted between the years of 2010 and 2011 with 6751 elderly individuals in 59 cities in the state of Rio Grande do Sul. The association between falling and the independent variables was verified by univariate and multivariate analysis, to estimate chances of falling, adjusted for sex and age. The frequency of falls was 10.7\%. Factors detected to be effective against falling: being male, having higher education, perception of insecurity, placing of seats in public places and participation in social and civic activities. The following were considered risk factors: old age, use of walking aids and participation in community activities. The results remained significant after adjustment for demographic factors. Falling is commonly associated with physical and behavioral aspects, though social and environmental factors also prove significant in this respect.
\end{abstract}

Key words Aging, Social participation, Community participation, Civic participation, Accidental falls, Risk factors
Resumo O objetivo deste artigo é investigar aspectos socioambientais associados às quedas em idosos residentes no estado do Rio Grande do Sul. Estudo descritivo, transversal, populacional, de base domiciliar, com coleta de dados primários, pertencente a um inquérito epidemiológico realizado no período de 2010 a 2011, com 6751 idosos de 59 cidades do estado do Rio Grande do Sul, Brasil, denominado Perfil dos Idosos do RS. A associação entre o desfecho quedas e as variáveis independentes foram analisadas de forma univariada e múltipla, através de regressão logística, calculando desta forma a chance associada à queda, ajustando por sexo e idade. A frequência de quedas foi de 10,7\%. Observamos serem fatores protetores: sexo masculino, maior escolaridade, percepção de insegurança e falta de bancos em locais públicos, participação em atividades sociais e cívicas. Foram considerados fatores de risco: faixa etária mais elevada, uso de órtese e participação em atividades comunitárias. Os resultados mantiveram-se significativos nas análises ajustadas. As quedas são comumente associadas a aspectos físicos e comportamentais, no entanto, as variáveis socioambientais também demonstram ser significativas nesse processo.

Palavras-chave Envelhecimento, Participação social, Participação comunitária, Participação cívica, Quedas, Fatores de risco 


\section{Introdução}

Embora o envelhecimento populacional seja conhecidamente um fenômeno mundial, ele ocorre de forma mais rápida e abrupta particularmente nos países em desenvolvimento, como o Brasil ${ }^{1}$.

Segundo o Censo 2010, a população idosa (60 anos ou mais de idade) era de 20,6 milhões de pessoas, contra 14,5 milhões em 2000, representando um crescimento de $41,7 \%$ contra $12,3 \%$ na população adulta. Por isso, o peso relativo da população idosa cresceu de $8,6 \%$ em 2000 para $10,8 \%$ em $2010^{2,3}$.

Nesse sentido, essa transição demográfica demonstra a necessidade de novas políticas públicas voltadas ao idoso em todas as esferas sociais, direcionando sua atenção às peculiaridades desse contingente populacional, assim como a avaliação do contexto no qual esta faixa etária está inserida ${ }^{4}$. Pensando nisso, a Organização Mundial de Saúde (OMS), realizou um estudo em 39 cidades do mundo sobre as principais dificuldades enfrentadas pelos idosos em diversos quesitos, tais como: habitação, transporte, locais públicos e serviços de saúde ${ }^{5}$. Baseado nesse estudo foi elaborada uma pesquisa de caráter quantitativo que buscou mensurar suas observações, bem como as características de saúde dos idosos do Rio Grande do Sul.

Dentre essas particularidades, destacam-se as quedas, uma vez que constituem o mais sério e frequente acidente que ocorre com idosos e a principal etiologia de morte acidental em pessoas desta faixa etária ${ }^{1,6}$. Por definição, a queda é caracterizada por um deslocamento não intencional do corpo para um nível inferior à posição inicial com incapacidade de correção em tempo hábil, determinado por circunstâncias multifatoriais comprometendo a estabilidade ${ }^{3}$.

As quedas e suas consequentes lesões estão associadas à diminuição da qualidade de vida ${ }^{7} \mathrm{e}$ ao aumento da morbimortalidade dos idosos, por isso constituem um problema de saúde pública de grande impacto social e econômico. Apesar do forte impacto dos fatores intrínsecos e domésticos descritos na literatura ${ }^{8}$, as quedas também podem ser influenciadas por fatores sociais. O estudo destes, por serem menos pesquisados que os fatores ambientais, e sua associação com o desfecho, é imprescindível para a detecção de fatores de risco e a prevenção".

O presente estudo tem por objetivo investigar aspectos socioambientais que possam estar associados a quedas em idosos residentes no estado do Rio Grande do Sul, um dos estados bra- sileiros com maior número de pessoas nessa faixa etária².

\section{Métodos}

Este estudo é parte do inquérito epidemiológico realizado no período de 2010 a 2011, com 7315 idosos de 59 cidades do estado do Rio Grande do Sul, Brasil, denominado Perfil dos Idosos do RS, realizado pelo Instituto de Geriatria e Gerontologia da Pontifícia Universidade Católica do Rio Grande do Sul (IGG-PUCRS) em parceria com a Escola de Saúde Pública do Rio Grande do Sul (ESP/RS).

O estudo foi baseado no "Guia Global: Cidade Amiga do Idoso", publicado pela $\mathrm{OMS}^{5}$, e objetivou identificar e avaliar o perfil dos idosos e o ambiente onde vivem, através de uma pesquisa representativa dessa faixa etária no Estado.

Este estudo caracterizou-se, assim, por ser descritivo, populacional, de base domiciliar, de corte transversal e com coleta de dados primários. A população a ser estudada foi composta por idosos de ambos os gêneros e residentes em domicílios do Estado do Rio Grande do Sul.

Para a seleção aleatória dos domicílios, obteve-se, junto ao Instituto Brasileiro de Geografia e Estatística (IBGE), a relação dos setores censitários de cada município escolhidos pelo sorteio nas macrorregiões do Estado. Em seguida procedeu-se a escolha dos setores, utilizando-se uma tabela de números aleatórios, sendo realizadas dezesseis entrevistas por setor.

Com a finalidade de obter-se uma representatividade de aleatoriedade, optou-se por adaptação da amostragem sistemática na seleção dos domicílios. Utilizou-se um croqui com os quarteirões numerados de cada setor censitário. $\mathrm{O}$ ponto de início do percurso, assim como a esquina inicial de cada quarteirão, foi sorteado, caminhando-se sempre no sentido horário. $\mathrm{Na}$ ausência de idoso ou idosa na residência selecionada, a seguinte foi visitada e assim sucessivamente até se conseguir realizar uma avaliação. Após o idoso ou idosa ter sido entrevistado(a), houve um pulo sistemático de oito casas, à partir da qual, iniciou-se a identificação de outro participante. O instrumento de pesquisa foi desenvolvido através do programa TeleForm ${ }^{\circledR} \mathrm{e}$ os dados foram coletados através de uma empresa prestadora de serviços. O instrumento de avaliação foi composto por 72 questões fechadas com escolha simples ou múltiplas, agrupadas em blocos temáticos que abordavam temas como: da- 
dos gerais do idoso, suas características e seu ambiente, transporte, renda, saúde, participação social, respeito e inclusão social, sexualidade, comunicação e informação, suporte comunitário e serviços de saúde e composição familiar. Foi realizado no início da entrevista um teste de evocação de três palavras (irmão, chave e avião). No caso dos idosos que não conseguiram repetir uma ou nenhuma palavra o questionário foi respondido pelo cuidador ou familiar.

Para esta análise, o desfecho utilizado foi a ocorrência de quedas no último ano, e as variáveis associadas foram: sexo, faixa etária, escolaridade, saiu de casa na última semana, percepção de locais públicos quanto à segurança, iluminação, presença de degraus e falta de bancos públicos, uso de órtese para locomoção, participação em atividades comunitárias (organizadas pela prefeitura, igreja, entre outros) e sociais (festas, shows, bailes e outros eventos festivos), conversa com amigos, participação em trabalho remunerado nos últimos seis meses e participação cívica do idoso (voto na última eleição). Foram incluídos apenas idosos que deambulavam de forma independente ou com auxílio de órteses (andador ou bengala). Desta forma, foram excluídos 564 idosos acamados e cadeirantes, pois os mesmos apresentam riscos diferenciados de queda, totalizando assim, 6751 analisados.

A variável idade foi agrupada por décadas, a partir dos 60 até 90 ou mais anos de idade. Tabelas de distribuição foram construídas cruzando o desfecho quedas e as variáveis independentes, as quais foram testadas pelo Qui-Quadrado.

A associação entre o desfecho e as variáveis independentes foram analisadas de forma múltipla por meio de regressão logística. Nesta análise incluímos somente as variáveis significativas no teste do Qui-Quadrado, calculando desta forma a chance associada à queda. Os modelos finais ajustados de regressão logística foram alcançados através da metodologia de retirada sistemática das variáveis menos significativas, a partir do modelo completo que incluía todas as variáveis independentes do estudo. Foram consideradas significativas as variáveis com associação que obtiveram nível de significância menor do que 0,05 . Os dados foram analisados por meio de programa EPI INFO 3.5.3.

O projeto de pesquisa foi aprovado pelo Comitê de Ética em Pesquisa da ESP/RS e da PUCRS, cumprindo integralmente os princípios éticos contidos na declaração de Helsinki, além do atendimento à legislação específica deste país.

\section{Resultados}

A idade dos 6751 idosos incluídos nesta análise variou de 60 a 102 anos, com uma média de 70,3 $\operatorname{anos}( \pm 7,3), 51,7 \%$ da amostra pertencia ao sexo feminino, $10,7 \%$ dos idosos referiram ao menos um episódio de queda, sendo $11,8 \%$ no sexo feminino e $9,4 \%$ no sexo masculino $(p=0,001)$.

A distribuição dos idosos que caíram ou não no último ano conforme as características sociodemográficas e a dificuldade de locomoção é apresentada na Tabela 1. Na Tabela 2 observa-se a distribuição desses idosos conforme a percepção sobre os locais públicos e na Tabela 3 a distribuição conforme a participação social e comunitária.

Foi verificado que $611(9,4 \%)$ idosos eram analfabetos, 1539 (23,6\%) foram alfabetizados e/ou estudaram até quatro anos incompletos, $2593(39,8 \%)$ completaram o ensino primário, 1086 (16,7\%) completaram o ginásio, 425 (6,5\%) estudaram até o ensino médio/técnico ou superior incompleto e apenas $266(4,1 \%)$ dos idosos completaram o ensino superior. Quando perguntado se haviam saído de casa na última semana, $5911(89,4 \%)$ entrevistados responderam que sim. Ao analisar a percepção deles em relação aos locais públicos, constatou-se que 1707 $(25,5 \%)$ idosos os consideraram inseguros, 602 $(8,9 \%)$ avaliaram como mal iluminados, 1303 $(19,3 \%)$ relataram a presença de poucos bancos e 2055 (30,4\%) não observaram problemas nesses aspectos. Em relação à deambulação, 6215(92,1\%) dos entrevistados relataram não necessitar de auxílio.

Quando perguntados sobre a participação em atividades comunitárias e sociais, 1204 (17,8\%) idosos afirmaram participar de atividades comunitárias ou desenvolvidas pelas prefeituras, envolvendo interação com outras faixas etárias e 2868 (41,6\%) entrevistados participavam de atividades sociais como festas, shows, bailes, almoços, entre outros eventos recreativos. Em relação à participação cívica, 4861 (72,0\%) idosos votaram nas últimas eleições, 5855 (86,7\%) conversaram com amigos que não moravam com ele na última semana e 5199 (89,0\%) não participaram de trabalho remunerado nos últimos seis meses.

Apenas as variáveis independentes: percepção de muitos degraus em locais públicos, frequência que conversa com amigos e participação em trabalho remunerado não foram significativamente associadas com o desfecho queda no último ano.

Os modelos finais ajustados de regressão logística foram alcançados através da metodolo- 
Tabela 1. Distribuição dos idosos conforme características sociodemográficas e dificuldade de locomoção entre os que caíram ou não no último ano.

\begin{tabular}{|c|c|c|c|c|c|}
\hline & \multicolumn{2}{|c|}{ Sem quedas } & \multicolumn{2}{|c|}{ Com quedas } & \multirow{2}{*}{ Valor $\mathbf{p}$} \\
\hline & $\mathbf{N}$ & $\%$ & $\mathbf{N}$ & $\%$ & \\
\hline Sexo & & & & & $\mathrm{p}=0,001$ \\
\hline Feminino & 3081 & 88,2 & 413 & 11,8 & \\
\hline Masculino & 2951 & 90,6 & 306 & 9,4 & \\
\hline Escolaridade & & & & & $\mathrm{p}<0,001$ \\
\hline Analfabeto & 525 & 85,9 & 86 & 14,1 & \\
\hline Alfabetizado & 1345 & 87,4 & 194 & 12,6 & \\
\hline Primário & 2294 & 88,4 & 299 & 11,6 & \\
\hline Ginásio & 1011 & 93,1 & 75 & 6,9 & \\
\hline Médio & 393 & 92,5 & 32 & 7,5 & \\
\hline Superior & 251 & 94,4 & 15 & 5,6 & \\
\hline Faixa Etária & & & & & $\mathrm{p}<0,001$ \\
\hline $60-69$ anos & 3340 & 93,6 & 227 & 6,4 & \\
\hline $70-79$ anos & 2010 & 86,6 & 312 & 13,4 & \\
\hline $80-89$ anos & 636 & 79,2 & 167 & 20,8 & \\
\hline 90 anos ou mais & 46 & 78 & 13 & 22 & \\
\hline Uso de Órteses & & & & & $\mathrm{p}<0,001$ \\
\hline Bengala ou andador & 356 & 66,4 & 180 & 33,6 & \\
\hline Nenhum Aparelho & 5676 & 91,3 & 539 & 8,7 & \\
\hline
\end{tabular}

Fonte: Perfil dos Idosos RS, 2010.

Tabela 2. Distribuição dos idosos conforme a percepção sobre os locais públicos entre os que caíram ou não no último ano.

\begin{tabular}{|c|c|c|c|c|c|}
\hline & \multicolumn{2}{|c|}{ Sem quedas } & \multicolumn{2}{|c|}{ Com quedas } & \multirow{2}{*}{ Valor p } \\
\hline & $\mathbf{N}$ & $\%$ & $\mathbf{N}$ & $\%$ & \\
\hline Inseguros & & & & & $\mathrm{p}<0,001$ \\
\hline Sim & 1573 & 92,1 & 134 & 7,9 & \\
\hline Não & 4457 & 88,4 & 585 & 11,6 & \\
\hline Pouco Iluminados & & & & & $\mathrm{p}=0,035$ \\
\hline Sim & 553 & 91,9 & 49 & 8,1 & \\
\hline Não & 5479 & 89,1 & 670 & 10,9 & \\
\hline Muitos Degraus & & & & & $\mathrm{p}=0,490$ \\
\hline Sim & 136 & 89,5 & 16 & 10,5 & \\
\hline Não & 5896 & 89,3 & 703 & 10,7 & \\
\hline Poucos Bancos & & & & & $\mathrm{p}<0,001$ \\
\hline $\operatorname{Sim}$ & 1226 & 94,1 & 77 & 5,9 & \\
\hline Não & 4806 & 88,2 & 642 & 11,8 & \\
\hline Não Observam Problemas & & & & & $\mathrm{p}=0,001$ \\
\hline Sim & 1592 & 90,1 & 463 & 9,9 & \\
\hline Não & 4440 & 87,5 & 256 & 12,5 & \\
\hline
\end{tabular}

Fonte: Perfil dos Idosos RS, 2010.

gia de retirada sistemática das variáveis menos significativas, a partir do modelo completo que incluía todas as variáveis independentes do estudo. Dessa maneira, para o desfecho, mantiveram-se significativas as variáveis da Tabela 4 . Níveis não significativos das variáveis: faixa etá- ria e escolaridade foram mantidos para preservar as comparações significativas observadas.

Idosos do sexo masculino apresentaram uma proteção de $21 \%$ para ocorrência de quedas ( $\mathrm{p}=$ $0,008)$. Em relação à escolaridade, idosos com nível superior apresentaram uma proteção de 
Tabela 3. Distribuição dos idosos conforme a participação social e comunitária entre os que caíram ou não no último ano.

\begin{tabular}{|c|c|c|c|c|c|}
\hline & \multicolumn{2}{|c|}{ Sem quedas } & \multicolumn{2}{|c|}{ Com quedas } & \multirow{2}{*}{ Valor $\mathbf{p}$} \\
\hline & $\mathbf{N}$ & $\%$ & $\mathbf{N}$ & $\%$ & \\
\hline Saiu de Casa & & & & & $\mathrm{p}<0,001$ \\
\hline $\operatorname{Sim}$ & 5328 & 90,1 & 583 & 9,9 & \\
\hline Não & 579 & 82,6 & 122 & 17,4 & \\
\hline Atividades Comunitárias & & & & & $\mathrm{p}<0,001$ \\
\hline Sim & 1040 & 86,4 & 164 & 13,6 & \\
\hline Não & 4992 & 90 & 555 & 10,0 & \\
\hline Atividades Sociais & & & & & $\mathrm{p}=0,005$ \\
\hline Sim & 2543 & 90,6 & 265 & 9,4 & \\
\hline Não & 3489 & 88,5 & 454 & 11,5 & \\
\hline Participação Cívica & & & & & $\mathrm{p}<0,001$ \\
\hline Sim & 4496 & 92,5 & 365 & 7,5 & \\
\hline Não & 1536 & 81,2 & 354 & 18,8 & \\
\hline Conversa com Amigos & & & & & $\mathrm{p}=0,902$ \\
\hline $\operatorname{Sim}$ & 5230 & 89,3 & 625 & 10,7 & \\
\hline Não & 802 & 89,5 & 94 & 10,5 & \\
\hline Trabalho Remunerado & & & & & $\mathrm{p}=0,166$ \\
\hline Sim & 671 & 90,7 & 69 & 9,3 & \\
\hline Não & 4668 & 89,8 & 531 & 10,2 & \\
\hline Recusa-se & 510 & 87,6 & 72 & 12,4 & \\
\hline
\end{tabular}

Fonte: Perfil dos Idosos RS, 2010.

$50 \%$ para o desfecho quando comparados com aqueles apenas alfabetizados. As chances de desenvolver o desfecho foram maiores na comparação de idade por década de vida entre as categorias de 80 a 89 anos e 60 a 69 anos $(\mathrm{p}<0,001)$.

Idosos necessitados de acessórios de auxílio para marcha apresentaram 4,12 vezes mais chance de sofrerem queda no último ano $(p<0,001)$.

Quando analisada a percepção de locais públicos, idosos que os classificaram como inseguros e com poucos bancos apresentaram proteção para o desfecho quedas $(\mathrm{p}<0,001)$, sendo $32 \%$ menor risco de quedas para ambientes inseguros e $44 \%$ para poucos bancos públicos. Aqueles que participavam de atividades comunitárias apresentaram 2,28 vezes mais chance de cair ( $\mathrm{p}<0,001$ ), já os idosos que participavam de atividades sociais tiveram proteção de $20 \%$ para quedas ( $\mathrm{p}=0,025$ ) e os que votaram nas últimas eleições apresentaram uma chance $36 \%$ menor de cair $(\mathrm{p}<0,001)$.

\section{Discussão}

Em relação à ocorrência de queda no último ano, o presente estudo observou uma frequência me-
Tabela 4. Modelo final de regressão logística do desfecho queda no último ano, Rio Grande do Sul, Brasil, 2010.

\begin{tabular}{lcr}
\hline \multicolumn{1}{c}{ Variáveis Independentes } & $\begin{array}{c}\text { Razão de } \\
\text { Chances }\end{array}$ & p \\
\hline Sexo (masculino / feminino) & $\underline{0,79}$ & $\underline{\underline{0,008}}$ \\
Faixa etária $(70-79 / 60-69)$ & $\underline{\underline{2,67}}$ & $\leq 0,001$ \\
Faixa etária $(80-89 / 60-69)$ & $\underline{2,21}$ & $\leq 0,001$ \\
Faixa etária (90 anos +/60 - 69) & 1,64 & 0,163 \\
Escolaridade (analfabeto/alfabetizado) & 0,84 & 0,270 \\
Escolaridade (primário/alfabetizado) & 1,02 & 0,831 \\
Escolaridade (ginásio/alfabetizado) & $\underline{0,70}$ & $\underline{0,020}$ \\
Escolaridade (médio/alfabetizado) & 0,80 & 0,305 \\
Escolaridade (superior/alfabetizado) & $\underline{0,50}$ & $\underline{0,017}$ \\
Uso de órtese (sim/não) & $\underline{4,12}$ & $\leq 0,001$ \\
Percepção local inseguro & $\underline{0,68}$ & $\leq 0,001$ \\
Percepção poucos bancos & $\underline{0,56}$ & $\leq 0,001$ \\
Ativ.comunitárias (sim/não) & $\underline{\underline{2,28}}$ & $\leq \underline{0,001}$ \\
Ativ.sociais (sim/não) & $\underline{0,80}$ & $\underline{0,025}$ \\
Participação cívica (sim/não) & $\underline{0,64}$ & $\leq \underline{0,001}$ \\
\hline
\end{tabular}

Fonte: Perfil dos Idosos RS, 2010.

nor do desfecho em idosos residentes na comunidade em comparação a outros estudos recentes, onde a frequência deste evento foi maior do que $30 \%{ }^{1,10,11}$. Já na análise por década de vida, 
as frequências variaram de 6,4-22\%. Essa proporção foi menor quando comparada com estudos internacionais que sustentam frequências de quedas variando $28-35 \%{ }^{12-14}$. Esse fato pode estar relacionado à diferença na metodologia utilizada ou ao delineamento dos estudos.

Em nosso estudo, idosos do sexo masculino apresentaram uma proteção de $21 \%$ para ocorrência de quedas. Nossos achados corroboram com outros estudos ${ }^{10,15,16}$ que relacionam o fato das mulheres serem mais acometidas por doenças crônico-degenerativas ao uso de maior número de medicamentos.

Em relação à variável escolaridade, observamos que idosos com mais anos de estudo apresentaram um menor risco de cair, o que se manteve significativo após a análise ajustada. Esta situação pode estar relacionada ao fato de que idosos com maior escolaridade tendem a ter uma maior renda e, consequentemente, melhores condições de moradia e acesso à saúde. Apenas um estudo com resultados semelhantes foi encontrado, o qual foi realizado com mais de 4000 idosos residentes na cidade de Pelotas (RS) e encontrou associação entre o desfecho e essa variável independente, no entanto, após a análise ajustada à associação manteve-se apenas com indicativo de significância ${ }^{11}$. Outro achado evidenciou uma maior frequência de quedas em idosos com baixa escolaridade apenas na análise não ajustada ${ }^{10}$.

A confiança que os idosos depositam em suas cidades é determinante em suas relações sociais e interações cotidianas, as quais ampliam a vida urbana e tendem a lhes garantir um envelhecimento ativo ${ }^{16}$. Deste modo, a variável percepção de locais públicos inseguros apresentou proteção contra a ocorrência de quedas, uma vez que esses idosos podem ter maior cuidado ao deambular nestes locais. A variável percepção de poucos bancos em locais públicos está relacionada com a proteção encontrada, pois provavelmente esses idosos apresentam maior limitação funcional e tendem a sair menos do seu domicílio. Outros estudos corroboram o fato de que na ausência de infraestrutura adequada, muitos idosos se limitam a realizar atividades nas ruas, especialmente os mais vulneráveis ${ }^{17,18}$.

Conforme os resultados encontrados, a chance de cair foi maior em indivíduos que utilizavam auxiliares de marcha. Diversos autores também encontraram resultados semelhantes ${ }^{6,19,20}$. Logo, a relação entre quedas e o uso de auxiliares para marcha deve-se, provavelmente, ao fato de que a necessidade de ajuda para locomoção, está, geralmente, associada a um maior comprometi- mento do padrão de marcha e dos mecanismos de controle postural, podendo ser considerado um indicador da fragilidade do idoso ${ }^{21}$.

Observamos também que os idosos que possuíam uma atividade social apresentaram um menor risco de quedas, ou seja, essa boa participação pode ter servido como um fator protetor. Outro estudo demonstrou que aqueles idosos com histórico de quedas múltiplas tendem a evitar atividades sociais quando comparados aos que não as sofreram ${ }^{22}$.

Além disso, um estudo longitudinal realizado com mulheres idosas que sofreram quedas, e como consequência a fratura de quadril, observou que durante $\mathrm{o}$ ano pós-fratura a função social melhorou, ou seja, a incapacidade do membro inferior não foi o fator limitante para a realização das atividades sociais. Esse mesmo estudo observou que os idosos que caíram mais de uma vez, tiveram menor participação nas atividades sociais no ano pós-fratura de quadril, do que aqueles idosos que não sofreram queda ${ }^{23}$.

A variável participação em atividades comunitárias foi considerada como fator de risco de para quedas. Estes resultados podem indicar que as pessoas participantes de algum tipo de atividade saem mais de casa, dessa forma ficando mais expostas às barreiras arquitetônicas. Fatores do ambiente como iluminação inadequada, superfícies escorregadias, degraus altos, ausência de corrimãos, calçadas inadequadas e vias públicas mal conservadas estão diretamente ligados ao aumento da chance de quedas ${ }^{24}$.

$\mathrm{Na}$ análise sobre a participação cívica e a chance de cair, não foram encontrados outros resultados que corroborem ou contradigam os achados desse estudo. Uma possível explicação seria o fato de que idosos que votam, exercendo seu papel cívico, na maioria das vezes talvez por apresentarem condições físicas de sair de casa, portanto apresentam maior capacidade funcional.

Uma das limitações deste estudo foi o uso de dados secundários, os quais limitaram a análise de algumas variáveis que seriam importantes em um estudo sobre quedas, como por exemplo, a avaliação de riscos domiciliares e a abordagem do local da queda. Além disso, este estudo teve delineamento transversal, o qual está sujeito ao efeito de causalidade reversa e limita o acompanhamento dos participantes, baseando-se em evidencias retrospectivas e de autorrelato. Sabe-se que em relação às quedas os idosos podem omitir esses eventos por esquecimento, vergonha, medo ou menosprezar a gravidade de uma queda, configurando mais uma limitação do estudo. 
É importante destacar o número reduzido de idosos que reportaram ter caído neste estudo. A literatura $^{6,10}$ sugere que $30 \%$ dos idosos caem a cada ano e neste estudo apenas 10,7\% dos participantes reportaram ter caído. Este fato pode ser explicado pela falta de uma definição específica para quedas, que pode ter levado os idosos a diferentes interpretações. Além disso, a maneira como a questão foi escrita, perguntando se o idoso "caiu no chão", também pode ter possibilitado diferentes interpretações. Outra possibilidade é que a maioria dos participantes deste estudo está na faixa etária de 60 a 69 anos. Talbot et al. ${ }^{9}$ encontraram que $21 \%$ dos adultos com idade entre 46 e 65 anos caíram nos dois anos em que o estudo foi realizado. $\mathrm{O}$ que demonstra que o risco de quedas aumenta com o avançar da idade. Além disso, estudos sugerem que idosos mais jovens podem ter quedas menos sérias ou se recuperar mais rapidamente das possíveis consequências quando comparados aos de mais idade ${ }^{25}$. Sendo assim, idosos mais jovens, como os participantes desta amostra, podem subestimar e não reportar as quedas.

As quedas são comumente associadas a aspectos físicos e comportamentais, no entanto, as variáveis socioambientais também demonstram ser significativas nesse processo. Uma readaptação do ambiente urbano, deixando-o mais seguro e atrativo para o idoso, torna-o mais propício para a vida comunitária.

Um melhor entendimento sobre esses fatores torna-se fundamental para a implementação de novas e mais abrangentes medidas públicas preventivas, contribuindo para a melhora da qualidade de vida do idoso e diminuindo também os recursos financeiros atualmente utilizados para o tratamento das consequências causadas pelas quedas.

\section{Colaboradores}

GN Pereira participou da redação final do artigo e da análise estatística; PM Morsch da redação final do artigo e ajustes pertinentes às normas para publicação; DGC Lopes, MD Trevisan, D Bós e MSS Vianna da redação final do artigo; A Ribeiro e JHN Navarro da redação final do artigo e tabulação dos dados; e AJG Bós da coleta de dados e análise estatística. 


\section{Referências}

1. Cruz DT, Ribeiro LC, Vieira MT, Teixeira MTB, Bastos RR, Leite ICB. Prevalência de quedas e fatores associados em idosos. Rev Saude Publica 2012; 46(1):138-146.

2. Instituto Brasileiro de Geografia e Estatística (IBGE). Perfil dos idosos responsáveis pelos domicílios no Brasil. Rio de Janeiro: IBGE; 2000.

3. Instituto Brasileiro de Geografia e Estatística (IBGE). Censo demográfico 2010. Rio de Janeiro: IBGE; 2011.

4. Rodrigues RAP, Kusumota L, Marques S, Fabricio SCC, Cruz IR, Lange C. Política nacional de atenção ao idoso e a contribuição da enfermagem. Texto Contexto Enferm 2007; 16(3):536-545.

5. Organização Mundial da Saúde OMS. Guia Global: Cidade Amiga do Idoso. Brasília: OMS; 2010.

6. Buksman S, Vilela ALS, Pereira SRM, Lino VS, Santos VH. Quedas em idosos: Prevenção. Projeto diretrizes. Brasília: Associação Médica Brasileira, Conselho federal de Medicina e Sociedade Brasileira de Geriatria e Gerontologia; 2008.

7. Ribeiro AP, de Souza ER, Atie S, de Souza AC, Schilithz AO. A influência das quedas na qualidade de vida de idosos. Cien Saude Colet 2008; 13(4):12651273.

8. Brasil. Ministério da Saúde (MS). Secretaria de Atenção à Saúde. Envelhecimento e saúde da pessoa idosa. Brasília: MS; 2006.

9. Talbot LA, Musiol RJ, Witham EK, Metter EJ. Falls in young, middle aged and older community dwelling adults: perceived caused, environmental factors and injury. BMC Public Health 2005; 5:86.

10. Perracini MR, Ramos LR. Fatores associados a quedas em uma coorte de idosos residentes na comunidade. Rev Saude Publica 2002; 36(6):709-716.

11. Siqueira FV, Facchini LA, Piccini RX, Tomasi E, Thumé E, Silveira DS, Vieira V, Hallal PC. Prevalência de quedas em idosos e fatores associados. Rev Saude Publica 2007; 41(5):749-756.

12. Davison J, Marrinan S. Falls. Rev Clin Gerontol 2007; 17(2):93-107.

13. Kenny RA, Romero-Ortuno R, Cogan L. Falls. Medicine 2009; 37(2):84-87.

14. Masud T, Morris RO. Epidemiology of falls. Age Ageing 2001; 30(4):3-7.

15. Aveiro MC, Driusso P, Barham EJ, Pavarini SC, Oishi J. Mobilidade e risco de quedas de população idosa da comunidade de São Carlos. Cien Saude Colet 2012; 17(9):2481-2488.
16. Bauman Z. Confiança e medo na cidade. Rio de Janeiro: Zahar; 2009.

17. Newton R, Ormerod M, Burton E, Mitchell L, WardThompson C. Increasing Independence for Older People through Good Street Design. IJIC 2010; 18(3): 24-29.

18. Hunter RH, Sykes K, Lowman SG, Duncan R, Satariano WA, Belza B. Environmental and Policy Change to Support Healthy Aging. J Aging Soc Pol 2011; 23(4):354-371.

19. Abreu SSE, Caldas CP. Velocidade de marcha, equilíbrio e idade: um estudo correlacional entre idosas participantes e não participantes de um programa de exercícios terapêuticos. Rev. Bras. Fisioter 2008; 12(4):324-330.

20. Carvalho AM, Coutinho ESF. Demência como fator de risco para fraturas graves em idosos. Rev Saude Publica 2002; 36(4):448-454.

21. Gama ZAS, Gómez-Conesa A. Factores de riesgo de caídas em ancianos: revisión sistemática. Rev Saude Publica 2008; 42(5):946-956.

22. Zijlstra GAR, Van Haastregt JCM, Van Eijk JTM, Van Rossum E, Stalenhoef PA, Kempen GIJM. Prevalence and correlates of fear of falling, and associated avoidance of activity in the general population of community-living older people. Age Ageing 2007; 36(3):304-309.

23. Miller RR, Ballew SH, Shardell MD, Hicks GE, Hawkes WG, Resnick B, Magaziner J. Repeat falls and the recovery of social participation in the year posthip fracture. Age Ageing 2009; 38(5):570-575.

24. Jahana KO, Diogo MJDE. Quedas em idosos: principais causas e consequências. Saúde Col 2007; 17(4):148-153.

25. Ruthig JC, Chipperfield JG, Newall NE, Perry RP, Hall NC. Detrimental effects of falling on health and well-being in later life: The mediating roles of perceived control and optimism. Journ Health Psych 2007; 12(2):231-248.

Artigo apresentado em 22/04/2013

Aprovado em 30/05/2013

Versão final apresentada em 14/06/2013 